\title{
Autonomic Dysfunction in Iraqi Patients with Chronic Inflammatory Demyelinating Polyneuropathy
}

\author{
Mohammed Majeed Kamil ${ }^{1}$, HaiderAli Mohammed ${ }^{2}$, Bahaa Hassan ${ }^{3}$, Basim Hanoon ${ }^{3}$, \\ Hassan Naji ${ }^{2}$ \\ ${ }^{1}$ Neurology Department, Baquba Teaching Hospital, Dyiala, Iraq \\ ${ }^{2}$ Neurology Department, Neurosurgery Teaching Hospital, Baghdad, Iraq \\ ${ }^{3}$ Neurology Department, Neurosciences Teaching Hospital, Baghdad, Iraq
}

Email address:

theneurologistbahaa@gmail.com (B. Hassan)

\section{To cite this article:}

Mohammed Majeed Kamil, Haidr Ali Mohammed, Bahaa Hassan, Basim Hanoon, Hassan Naji. Autonomic Dysfunction in Iraqi Patients with Chronic Inflammatory Demyelinating Polyneuropathy. American Journal of Internal Medicine.

Vol. 6, No. 1, 2018, pp. 10-19. doi: 10.11648/j.ajim.20180601.12

Received: December 6, 2017; Accepted: December 18, 2017; Published: January 29, 2018

\begin{abstract}
Objective:The purpose of this study is to evaluate the autonomic disturbance in patients with chronic inflammatory demyelinating polyneuropathy.Methods:Thirty-eight patients who fit into the AAN criteria of diagnosis of CIDP were studied. Five standard cardiovascular autonomic Ewing tests were performed for every patient in addition to the assessment of autonomic symptoms.Results: The study includes 32 males and 6 females their age ranged from 10 to 69 years. In this study we have seen one child with CIDP whose age was ten years and he had PST impairment. Sexual disturbance was the majorautonomic symptom seen in the males [40.6\%], while urinary disturbance 2 was the major autonomic disturbance encountered in females [66.6\%]. Autonomic function tests [AFT] showed that one quarter of males and half of the females had abnormal AFT. In patients aged between 50-59 years, [40\%] having normal autonomic function test, [40\%] had PST impairment, while the others have mixed impairment [20\%]. None of the patients had pure sympathetic impairment. Regarding the types of CIDP the largest percent [84.2\%] of patients have classic type of CIDP while 7.8\%, 5.2\% and 2.6\% had DADS, MADSAM and MMN respectively.Conclusions: Autonomic dysfunction was common in Iraqi CIDP patients. PST impairment is a major pattern of dysautonomia in CIDP. Sexual disturbance in males while urinary disturbance in females were the most common autonomic symptoms encountered. VM, HR response to standing allow an early detection of autonomic dysfunction even in asymptomatic individuals.
\end{abstract}

Keywords: Autonomic Dysfunction, CIDP, Iraqi Patients

\section{Introduction}

\subsection{Definition}

The term chronic inflammatory demyelinating polyradiculoneuropathy [CIDP] has been used to identify patients with a chronically progressive or relapsing symmetric sensorimotor disorder with cytoalbuminologic dissociation and interstitial and perivascular endoneurial infiltration by lymphocytes and macrophages. In many ways, CIDP can be considered the chronic equivalent of acute inflammatory demyelinating polyradiculoneuropathy [AIDP], the most common form of Guillain-Barré syndrome [GBS]. [1]

\subsection{Classification}

1- Classic CIDP:

Classic chronic inflammatory demyelinating polyradiculoneuropathy [CIDP] is characterized by the occurrence of symmetrical weakness in both proximal and distal muscles that progressively increases for more than two months. The condition is associated with impaired sensation, absent or diminished tendon reflexes, an elevated cerebrospinal fluid protein level, demyelinating nerveconduction studies, and signs of demyelination in nerve- 
biopsy specimens. The course can be relapsing or chronic and progressive, the former being much more common in young adults. [2-4]

2- Distal Acquired Demyelinating Symmetric Neuropathy [DADS]:

Autonomic Dysfunction in Patients with CIDP Introduction

It has been suggested that distal acquired demyelinating symmetric neuropathy is a distinct acquired demyelinating polyneuropathy. [5] Features of the disorder include an increased prevalence in men and in persons over the age of 50 years, a predominantly distal sensory loss, a mild distal weakness (as opposed to the more generalized motor deficits in classic chronic inflammatory demyelinating polyneuropathy), and an unsteady gait. IgMparaproteinemia is present in nearly two thirds of patients with this condition. [6] IgM-associated distal demyelinating symmetric neuropathy seems to respond poorly to immunosuppressive therapy. [6]

3- Multifocal Motor Neuropathy [MMN]:

Multifocal motor neuropathy is characterized by asymmetric weakness without sensory loss, often starting in distal arm muscles. A partial motor-conduction block at multiple sites is a characteristic electrophysiologicfeature, although not all patients have this finding. Cerebrospinalfluid protein levels and cell counts are usually normal. Although corticosteroids and plasmapheresis are ineffective treatments, multifocal motor neuropathy improves with immune globulin [7] or cyclophosphamide [8] therapy.

4- Multifocal Acquired Demyelinating Sensory and Motor Neuropathy the [Lewis-Sumner Syndrome]:

Multifocal acquired demyelinating sensory and motor neuropathy [the Lewis-Sumner syndrome] has similarities to both chronic inflammatory demyelinating polyneuropathy [i.e., motor and sensory deficits, an elevated protein content, and abnormal results on motor-nerve and sensorynerveconduction studies] and multifocal motor neuropathy [i.e., asymmetrical presentation of symptoms, often starting from the arms and hands, and conduction block].[9-11]. Some patients with the condition have antibodies to gangliosides, [12] and these patients have a reasonably good response to treatment with intravenous immune globulin or cyclophosphamide.

\subsection{Historical Background}

Eichhorst [13] first described recurrent polyneuritis in 1890. Sporadic case reports followed, including a case series by Hoestermann in 1914 that described a 50-year-old man who experienced 6 bouts of polyneuropathy between 1870 and 1900. A report from 1929 that describeda case of recurrent polyneuropathy in a 45-year-old man further demonstrates the cardinal clinical features of relatively symmetric proximal and distal weakness, hyporeflexia or areflexia, and to a lesser degree, sensory disturbances. [4]

Elevation of cerebrospinal fluid protein without pleocytosis[albuminocytologic dissociation] was reported in a case of relapsingpolyneuritis in 1931. Fifteen years earlier, Guillain, Barre', and Strohlhad reported this laboratory finding in 2 French soldiers with acute polyneuritis.[15]

The concept of segmental demyelination causing conduction block was not new, having been reported by Denny-Brown and Brenner[15] following experiments with tourniquet paralysis of the cat sciatic nerve. Segmental demyelination was later found to be the predominant histopathologic feature of the human sural nerve in cases of CIDP. [16]

In 1975, Dyck and colleagues proposed diagnostic criteria in a landmark publication that described 53 patients with "chronic inflammatory polyradiculoneuropathy." [17]

In 1958, Austin was separated CIDP from acute inflammatory polyradiculoneuropathy, or GBS, on the basis of a prolonged and relapsing course and responsiveness to corticosteroids. [17, 18]

\subsection{Epidemiology}

Chronic inflammatory demyelinating polyneuropathy is a common, albeit under diagnosed and potentially treatable disease with an estimatedprevalence of about 0.5 per 100,000 children [19] and 1 to 2 per 100,000 adults. [20, 21].

Other studies report a prevalence of $2-7.7 / 100,000$.[20, 22] This would correspond to approximately 5,000 to 22,000 cases in the United States.

This degree of variance underscores the difficulties associated with the diagnosis of CIDP.

Both sexes are affected equally. MMN has male-female predominance of at least 2:1 based on a survey of the largest case series. [23]

CIDP may occur at any age, but it is more common in the fifth and sixth decades. Relapsing course is associated with younger age of patients [third and fourth decades]. CIDP has been described in childhood. [24]

\subsection{Pathophysiology}

CIDP is presumed to occur because of immunologic antibody-mediated reaction along with interstitial and perivascular infiltration of theendoneurium with inflammatory $\mathrm{T}$ cells and macrophages. The consequence is a segmental demyelination of peripheral nerves. [25]

Human leukocyte antigens Dw3, DRw3, A1, and B8 occur more frequently in patients with CIDP than in the healthy population. [25]

\subsection{Etiology}

CIDP is most frequently an idiopathic illness, but it has been known to occur with several conditions. In those cases, the associated condition is included in the main diagnosis [for example, CIDP with paraproteinemia, CIDP with HIV infection] to separate those cases from the idiopathic variety Most reported conditions associated with CIDP are listed below:[26]

-HIV infection: In these patients, mild lymphocytic pleocytosis and increased gamma globulin level in the CSF are seen frequently.

-Hodgkin lymphoma. Neuropathy associated with 
Hodgkin lymphoma is not caused by direct infiltration of the peripheral nerves but is a consequence of the autoimmune cascade that occurs with this disease; the mechanism is not completely clear.

-Paraproteinemias and/or plasma cell dyscrasias

a-CIDP is seen with monoclonal gammopathies [eg, monoclonal gammopathy of unknown significance [MGUS]), most frequently gammopathy of immunoglobulin M [IgM]. Evidence exists to suggest that CIDP with IgM MGUS has specific clinical and electrophysiologic characteristics. Patients usually have predominance of distal weakness, for example, and sensory symptoms are greater than motor. [27]

b- Some paraproteinemias occur as isolated phenomena and some are by-products of malignant cells, as in the cases of Waldenströmmacroglobulinemia and myeloma. In myeloma-associated neuropathy, theabnormal paraprotein usually consists mostly of lambda light chain component. Combination of osteosclerotic myeloma, organomegaly, endocrinopathy, $\mathrm{M}$ protein, sensorimotor neuropathy, and pigmentary skin changes is referred to as POEMS syndrome. In POEMS syndrome, the $M$ protein is typically immunoglobulin G [IgG].

-Systemic lupus erythematosus.

-Inflammatory bowel disease: CIDP has been described in association with Crohn disease and other inflammatory bowel conditions, although no direct correlation between the 2 afflictions is known.

-Diabetes mellitus: Increasing evidence supports the suggestion that some patients with diabetes who have severe neuropathy or unusually progressive neuropathy may have CIDP superimposed on their diabetic disorder. [28, 29]

-Occasionally, chronic inflammatory demyelinating polyneuropathy may develop in a setting of another polyneuropathy, even one with ahereditary basis, such as Charcot-Marie-Tooth disease. [30]

\subsection{Clinical Presentation}

\subsubsection{History}

CIDP most frequently starts insidiously and evolves slowly, either in a slowly progressive [more than $60 \%$ of patients] or relapsing manner [approximately one third of patients], with partial or complete recovery between recurrences. Periods of worsening and improvement usually last weeks or months. Initial symptoms include weakness of the limbs, both proximal and distal, with proximal muscles affected at least as severely as distal. Sensory symptoms are common.

Only a small proportion of patients approximately $16 \%$ have a relatively acute or subacute onset of symptoms, with subsequent steadily progressive or fluctuating course.

Most experts consider the necessary duration of symptoms to be 8-12 weeks for the diagnosis of CIDP to be made.

\subsubsection{Physical examination}

Depending on the associated systemic disorder, abnormalities on physical examination may be found in multiple organ systems. Patients should be examined in detail for signs of autoimmune, inflammatory, and neoplastic conditions.

Cranial nerves:

Cranial nerves may be involved, particularly CN VII, with paralysis of both upper and lower facial muscles. Diplopia can occur with the involvement of CN III, IV, or VI. Rarely, bulbar muscles [e.g., palate, tongue] can be affected.

Papilledema with pseudotumorcerebri syndrome are observed rarely in patients with CIDP and are due to a very high CSF protein level

Gait:

Gait frequently is abnormal.

Motor system:

Usually relatively symmetric weakness of both proximal and distal muscles is present in upper and lower extremities. Muscle tone can be normal or decreased. Hypotonia, atrophy, and fasciculations may be present. Deep tendon reflexes characteristically are diminished or absent even in regions with only mild weakness.

Sensory system:

Large-diameter, heavily myelinated fibers are affected most severely, leading to proprioceptive and vibratory deficits. Loss or decrease of pain [i.e., pinprick] and temperature sensations is less common. Stocking-glove distribution of sensory deficits is typical. Neuropathic pain in affected extremities may occur. [3, 17]

\subsection{Neurotransmitters}

Acetylcholine [Ach] is the preganglionic neurotransmitter for both divisions of the ANS as well as the postganglionic neurotransmitter of PST neurons. Nerves that release Ach are said to be cholinergic.

Norepinephrine [NE] is a neurotransmitter of postganglionic ST neurons; these nerves are said to be adrenergic. Within the ST outflow, postganglionic neurons innervating the eccrine sweat glands [and perhaps some blood vessels supplying skeletal muscles] are of cholinergic type [38].

\subsection{The Functions of ANS}

The ANS innervates vascular and visceral smooth muscles, exocrine and endocrine glands and parenchymal cells throughout the various organ systems. Functioning below the conscious level, the ANS respond rapidly and continually to perturbations that threaten the constancy of the internal environment.

The many functions governed by this system include the distribution of blood flow and the maintenance of tissue perfusion, the regulation of blood pressure, the regulation of volume and composition of extracellular fluid, the expenditure of metabolic energy and supplying substrate, and control of visceral smooth muscles and glands [38, 39].

\subsection{Autonomic Testing}

The functional characteristics of ANS can be assessed by physiologic and pharmacological tests. When autonomic neuropathy is suspected, non-invasive tests can be used initially to confirm the diagnosis, and to determine whether 
ST or PST pathways, or both are involved.

In some cases further studies requiring intra-arterial catheterization may be needed to localize the site of the lesion in ANS more precisely [35, 40], commonly used physiologic tests primarily assess the autonomic aspects of cardiovascular functioning. These tests are non-invasive, easy to use and provide quantitative or regional information about autonomic functioning.

Interpretation of results requires collection of data under controlled circumstances. In addition to five tests described by Ewing \& Clarke [41], other tests are available, like cold pressure test, in which subjects immerse his hand in ice water and after one minute systolic and diastolic blood pressure normally rise by $10-20 \mathrm{mmHg}$.

Sudomotor functioning tests are used to assess the STpostganglionic fibers either as quantitative test [quantitative sudomotor axon reflex test] or as qualitative measure [thermo regulating sweat test] $[42,43]$.

The pharmacological tests elucidate pathophysiological abnormalities, help to localize an autonomic defect to the central or peripheral NS and guide the development of rational therapy, for example, measuring of nor epinephrine levels in response to standing or by using drugs with presser activity e.g. Tyramine [35, 40, 42].

\section{Aims of the Study}

The aim of the study was to investigate:

1-The frequency of autonomic dysfunction in Iraqi CIDP patients.

2-The distribution of autonomic dysfunction in relation to age, sex and the types of CIDP

\section{Subjects and Methods}

Thirty-eight patients who fit into the AAN criteria of diagnosis of CIDP [4] were studied during the period between June 20015 and April 20016 in the Department of Neurology at, neurosciences hospital,baquba teaching hospital and neurosurgery hospital Baghdad Iraq.

The patients were diagnosed as clinically definite CIDP. Each patient was interviewed and assessed according to a protocol paper [appendix 1].

Every patient was fully examined neurologically, medically and electro physiologically. Cerebrospinal fluid was aspirated for every patient.

The patients were on no medications known to affect the autonomic functions. They are divided into six groups according to age, into two groups according to gender and into four groups according to the types of CIDP.

Five standard cardiovascular Ewing tests [41], were performed for every patient as shown in appendix 2, and these include:

1-Heart rate response to Valsalva maneuver [VM].

2-Heart rate [R-R interval] variation during deep breathing.

3-Immediate heart rate response to standing.

4-Blood pressure response to standing.

5-Blood pressure response to isometric handgrip.

Data were presented with simple measures of frequency and percentages.

\section{Results}

The study was performed on thirty eight clinically definite CIDP patients. In patient groups there were males and females.

Table 1. Characteristics of the study Subjects: This table divided the study subjects according to age into six groups, $34.2 \%$ of them are lying in 20-29 category and they are males. Total number of males is five times more than thetotal number of females.

\begin{tabular}{llll}
\hline total & No. of females & No. of males & Age group \\
\hline $1(2.6 \%)$ & 0 & 1 & $10-19$ years \\
$13(34.2 \%)$ & 0 & 13 & $20-29$ years \\
$9(23.6 \%)$ & 2 & 7 & $30-39$ years \\
$8(21 \%)$ & 1 & 7 & $40-49$ years \\
$5(13.1 \%)$ & 1 & 4 & $50-59$ years \\
$2(5.2 \%)$ & 2 & 0 & $60-69$ years \\
38 & 6 & 32 & total \\
\hline
\end{tabular}

Table 2. Distribution of autonomic symptoms in patients with CIDP: The percents of CV, urinary, GIT and sweating disturbances are higher in females than the males and the percents of GIT and urinary disturbances are equal in total subjects. $40.6 \%$ of males had sexual disturbances.

\begin{tabular}{llll}
\hline Total no. (\%) & Females no. (\%) & Males no. (\%) & Autonomic symptoms \\
\hline $4(10.5 \%)$ & $3(50 \%)$ & $1(3.1 \%)$ & CV disturbance \\
$6(15.7 \%)$ & $4(66.6 \%)$ & $2(6.2 \%)$ & Urinary disturbance \\
$6(15.7 \%)$ & $2(33.3 \%)$ & $4(12.5 \%)$ & GIT disturbance \\
$13(34.2 \%)$ & $/$ & $13(40.6 \%)$ & Sexual disturbance \\
$2(5.2 \%)$ & $1(16.6 \%)$ & $1(3.1 \%)$ & Sweating disturbance \\
\hline
\end{tabular}

Table 3. The distribution of CV autonomic symptoms among patients with CIDP: CV symptoms were presented mostly in females, while just 3.1\% of males had palpitation, and the others are symptoms free.

\begin{tabular}{llll}
\hline Total no. $(\%)$ & Females no. $(\%)$ & Males no. (\%) & CV autonomic symptoms \\
\hline $2(5.2 \%)$ & $2(33.3 \%)$ & $0(0 \%)$ & Postural dizziness \\
$0(0 \%)$ & $0(0 \%)$ & $0(0 \%)$ & syncope \\
$2(5.2 \%)$ & $1(16.6)$ & $1(3.1 \%)$ & palpitation \\
$1(2.6 \%)$ & $1(16.6)$ & $0(0 \%)$ & Flushing sensation \\
\hline
\end{tabular}


Table 4. The distribution of sexual dysfunction symptoms among males with CIDP: The largest percent is of males having loss of early morning erection $(21.8 \%)$, while $15.6 \%$ of them having impotence and only small percent of them having impaired ejaculation.

\begin{tabular}{ll}
\hline No. $(\%)$ & sexual dysfunction symptom \\
\hline $5(15.6 \%)$ & Impotence \\
$1(3.1 \%)$ & Impaired ejaculation \\
$0(0 \%)$ & Loss of libido \\
$7(21.8 \%)$ & Loss of early morning erection \\
\hline
\end{tabular}

Table 5. The distribution of urinary symptoms among patients with CIDP: The percents of urinary symptoms in males are lower than that of females, only $6.2 \%$ of males had frequency while $33.3 \%$ of females had frequency, $16.6 \%$ had incontinence and $16.6 \%$ had nocturia. None of the patients had urgency or hesitancy.

\begin{tabular}{llll}
\hline Total no. (\%) & Female no. (\%) & Males no. (\%) & Urinary symptom \\
\hline $4(10.5 \%)$ & $2(33.3 \%)$ & $2(6.2 \%)$ & Frequency \\
$1(2.6 \%)$ & $1(16.6 \%)$ & $0(0 \%)$ & Incontinence \\
$1(2.6 \%)$ & $1(16.6 \%)$ & $0(0 \%)$ & Nocturia \\
$0(0 \%)$ & $0(0 \%)$ & $0(0 \%)$ & Urgency \\
$0(0 \%)$ & $0(0 \%)$ & $0(0 \%)$ & Hesitancy \\
\hline
\end{tabular}

Table 6. The distribution of GIT symptoms in patients with CIDP: Equal percents (3.1\%) of patients having constipation and those having dry mouth presented in males. $6.2 \%$ of males have dyspepsia. In females $16.6 \%$ have constipation and again 16.6\% have dry mouth.

\begin{tabular}{llll}
\hline Total no. (\%) & Female no. (\%) & Males no. (\%) & GIT symptom \\
\hline $2(6.2 \%)$ & $1(16.6 \%)$ & $1(3.1 \%)$ & Constipation \\
$0(0 \%)$ & $0(0 \%)$ & $0(0 \%)$ & Incontinence \\
$2(6.2 \%)$ & $0(0 \%)$ & $2(6.2 \%)$ & Dyspepsia \\
$2(6.2 \%)$ & $1(16.6 \%)$ & $1(3.1 \%)$ & Dry mouth \\
\hline
\end{tabular}

Table 7. The distribution of sweating disturbance among patients with CIDP: Only 5.2\% of the study subjects complained from dry skin while none of them complained from excessive sweating.

\begin{tabular}{ll}
\hline Patients no. (\%) & sweating disturbance \\
\hline $2(5.2 \%)$ & Dry skin \\
$0(0 \%)$ & Excessive sweating \\
\hline
\end{tabular}

Table 8. Autonomic function test in patients with CIDP: High percent of the study subjects have normal autonomic function test (71\%) and $21 \%$ of them have parasympathetic impairment. None of them had pure sympathetic impairment while $7.8 \%$ have mixed impairment.

\begin{tabular}{llll}
\hline Total no. (\%) & Females no. (\%) & Males no. (\%) & Autonomic function test \\
\hline $27(71 \%)$ & $3(50 \%)$ & $24(75 \%)$ & Normal \\
$8(21 \%)$ & $1(16.6 \%)$ & $7(21.8 \%)$ & Parasympathetic impairment \\
$0(0 \%)$ & $0(0 \%)$ & $0(0 \%)$ & Sympathetic impairment \\
$3(7.8 \%)$ & $2(33.3 \%)$ & $1(3.1 \%)$ & Mixed impairment \\
\hline
\end{tabular}

This table showed that $100 \%$ of patients aged between 10-19 years having parasympathetic impairment, $100 \%$ of patients aged between 30-39 years having normal autonomic function test and $100 \%$ of patients aged between 60-69 years having mixed impairment. $76.9 \%$ of patients aged between 20-29 years having normal autonomic function test while the others [23.7\%] having parasympathetic impairment. Within 40-49 age group; $75 \%$ of them having normal autonomic function test and $25 \%$ having parasympathetic impairment. Equal percents [40\%] of patients aged between 50-59 years having normal autonomic function test and parasympathetic impairment, while the others have mixed impairment [20\%].

Table 9. Autonomic function test in study subjects according to age.

\begin{tabular}{|c|c|c|c|c|c|}
\hline \multirow{2}{*}{ Mixed imp no. (\%) } & \multirow{2}{*}{ Symp imp no. (\%) } & \multirow{2}{*}{ Parasymp imp no. (\%) } & \multirow{2}{*}{$\begin{array}{l}\text { Normal } \\
\text { no. }(\%)\end{array}$} & \multirow{2}{*}{ No. } & \multirow{2}{*}{ Age group } \\
\hline & & & & & \\
\hline $0(0 \%)$ & $0(0 \%)$ & $1(100 \%)$ & $0(0 \%)$ & 1 & 10-19 years \\
\hline $0(0 \%)$ & $0(0 \%)$ & $3(23.7 \%)$ & $10(76.9)$ & 13 & $20-29$ years \\
\hline $0(0 \%)$ & $0(0 \%)$ & $0(0 \%)$ & $9(100 \%)$ & 9 & $30-39$ years \\
\hline $0(0 \%)$ & $0(0 \%)$ & $2(25 \%)$ & $6(75 \%)$ & 8 & $40-49$ years \\
\hline $1(20 \%)$ & $0(0 \%)$ & $2(40 \%)$ & $2(40 \%)$ & 5 & $50-59$ years \\
\hline $2(100 \%)$ & $0(0 \%)$ & $0(0 \%)$ & $0(0 \%)$ & 2 & $60-69$ years \\
\hline
\end{tabular}


Table 10. The distribution of autonomic function test result in patient with CIDP.

\begin{tabular}{llll}
\hline Patient no. $=38$ & \multicolumn{3}{c}{ Autonomic function test } \\
\hline Abnormal No. (\%) & Borderline No. $(\%)$ & Normal No. (\%) & \\
\hline $2(5.2 \%)$ & $0(0 \%)$ & $36(94.7 \%)$ & BP response to standing \\
$3(7.8 \%)$ & $0(0 \%)$ & $35(92.2 \%)$ & Isometric test \\
$3(7.8 \%)$ & $2(5.2 \%)$ & $33(86.8 \%)$ & R-R variation deep breathing \\
$5(13.1 \%)$ & $3(7.8 \%)$ & $30(78.9 \%)$ & Valsalva \\
$4(10.5 \%)$ & $2(5.2 \%)$ & $32(84.2 \%)$ & R-R variation on standing \\
\hline
\end{tabular}

Table 11. Types of CIDP: The largest percent (84.2\%) of patients have classic type of CIDP while $7.8 \%, 5.2 \%$ and $2.6 \%$ had DADS, MADSAM and MMN respectively.

\begin{tabular}{ll}
\hline No. $(\%)$ & Type of CIDP \\
\hline $32(84.2 \%)$ & Classic \\
$3(7.8 \%)$ & DADS \\
$2(5.3 \%)$ & MADSAM \\
$1(2.7 \%)$ & MMN \\
\hline
\end{tabular}

$100 \%$ of patients with MMN type have normal autonomic function test and complained from symptoms. One hundred percent of patients with MADSAM type have abnormal autonomic function test and also complained from symptoms.

Fifty percent of patients with classic type of CIDP presented with normal AFT but without symptoms, while $25 \%$ of them presented with normal AFT but with presence of symptoms, the rest are presented with abnormal AFT and complained of symptoms. $66.6 \%$ of DADS type patients presented with normal AFT and with presence of symptoms, while the rest presented with abnormal AFT but without symptoms.

Table 12. Distribution of AFT in relation to autonomic symptoms and the type of CIDP.

\begin{tabular}{|c|c|c|c|c|c|}
\hline \multicolumn{6}{|c|}{ Types of CIDP } \\
\hline Total $=38$ & MADSAMTotal no. $=2$ & MMNTotal no. $=1$ & DADSTotal no. $=3$ & ClassicTotal no. $=32$ & \\
\hline $11(28.9 \%)$ & $0(0 \%)$ & $1(100 \%)$ & $2(66.6 \%)$ & $8(25 \%)$ & Normal AFT with +ve symptoms \\
\hline $10(26.3 \%)$ & $2(100 \%)$ & $0(0 \%)$ & $0(0 \%)$ & $8(25 \%)$ & Abn. AFT with +ve symptoms \\
\hline $16(42.1 \%)$ & $0(0 \%)$ & $0(0 \%)$ & $0(0 \%)$ & $16(50 \%)$ & Normal AFT with -ve symptoms \\
\hline $1(2.6 \%)$ & $0(0 \%)$ & $0(0 \%)$ & $1(33.3 \%)$ & $0(0 \%)$ & Abn. AFT with -ve symptoms \\
\hline
\end{tabular}

Table 13. The distribution of autonomic symptoms and AFT according to sex: The percent of females presented with positive symptoms are higher than males (whether having normal or abnormal AFT), while the situation is reversed in case of patients with out symptoms.

\begin{tabular}{lll}
\hline Females no. $=\mathbf{6}$ & & Males no. $=\mathbf{3 2}$ \\
\hline $2[33.3 \%]$ & $9[28.1 \%]$ & Normal AFT with +ve symptoms \\
$3[50 \%]$ & $7[21.8 \%]$ & Abn. AFT with +ve symptoms \\
$1[16.6 \%]$ & $15[46.8 \%]$ & Normal AFT with -ve symptoms \\
$0[0 \%]$ & $1[3.1 \%]$ & Abn. AFT with -ve symptoms \\
\hline
\end{tabular}

\section{Discussion}

This is the first study investigating in details the autonomic dysfunction in Iraqi CIDP patients.

This study showed that the battery of ANS tests could be used in assessment of ANS abnormalities in CIDP patients, the most precise detection of autonomic reflexes can be achieved by using of the whole battery of tests.

In this study the total no. of males was five times more than the total no. of females; these findings did not match with other studies that showed that equal sex distribution in CIDP patients. [23]

The majority of male patients with CIDP were in the $3 \mathrm{rd}$ decade of their life while no reported cases have been seen in the 7 th decade. In contrast, third of females with CIDP were in the 7 th decade, while no reported cases have been seen in the 3rd decade.

CIDP has been described in childhood [24]; in this study we have seen one child with CIDP whose age was ten years.

This study showed that the symptoms related to autonomic disturbances in CIDP were present in the vast majority of patients, even at the onset of the disease and showed a difference in distribution between males and females.

Sexual disturbance was the main autonomic symptom in male patients with CIDP [40.6\%], and approximately half of those patients had loss of early morning erection, while urinary disturbance was the major autonomic symptom in female patients [66.6\%] followed by cardiovascular autonomic symptoms (half of the females).

In general, it's appeared that autonomic symptoms were more frequent in females than males apart from sexual disturbance.

The high frequency of autonomic symptoms found in this study should encourage the practicing clinician to look carefully for these abnormalities in the evaluation of the patients and not merely emphasize on the usual CIDP features, as these autonomic disturbances may be disabling and in most of the times, simple measures to overcome such disturbances may have great deal in helping the patients to improve their quality of life. 
Autonomic function tests [AFT] done according to Ewing $\&$ Clarke [41] showed that one quarter of males and half of the females had abnormal AFT.

Pure PST impairment was seen in $21 \%$ of the study subjects and it was slightly higher in males than in females. Pure ST impairment was not seen in the study subjects. Mixed PST and ST impairment were seen in both males and females but it is much higher in females in comparison to males (33.3\% in females and $3.1 \%$ in males).

Regarding the age of the patients; all patients aged between 10-19 years, 30-39 years and 60-69 years have PST impairment, normal AFT and mixed impairment respectively. At the age group between 20-29 years and 40-49 years; one quarter have PST impairment while the rest have normal AFT.

In patients aged between 50-59 years, [40\%] having normal autonomic function test, [40\%] had PST impairment, while the others have mixed impairment [20\%].

The heart rate response to $\mathrm{VM}$ and to immediate standing and blood pressure response to sustained handgrip had the disadvantage of being effort dependent, thus disabled patients may need help of others to overcome their disability. The cooperation of those disabled is essential to obtain satisfactory performance of the above tests. This fact should be consideredwhen $\mathrm{CV}$ reflexes are assessed. To overcome some of those disabilities, certain instruments have been developed to help in the assessment of the crippled patients.

A critical point in the assessment of autonomic functions was the age of the patients, as autonomic functions might be affected when the age advanced, this must be taken into consideration when the results are analyzed.

In this study, abnormal VM is the most common abnormality encountered followed by the heart rate variation on standing and then the heart rate variation on deep breathing. Also abnormal isometric test was more common than BP response to standing.

Regarding the types of CIDP, classic CIDP was a phenomenon being the most common type seen in this study $(84.2 \%)$, while the other types are much less [15.8\%]. Half of the patients with classic CIDP had normal AFT without autonomic symptoms; one quarter of them had also normal AFT but complained from symptoms such as loss of early morning erection. Towthird of patients with DADS had normal AFT and complained from autonomic symptoms while the rest onethird had abnormal AFT but they did not complained from autonomic symptoms. The patient with MMN had normal AFT but he complained from loss of early morning erection. All patients with MADSAM had abnormal AFT and complained from autonomic symptoms.

In this study, half of female patients had abnormal AFT and complained from autonomic symptoms, in contrast, approximately half of males patients had normal AFT with out complain of autonomic symptoms.

\section{Conclusions}

1- Autonomic dysfunction assessed by Ewing et. al. tests were common in Iraqi CIDP patients.

2- Dysregulation of PST cardiovascular control mechanism is a major pattern of dysautonomia in CIDP. Combined [ST and PST ] was less common in CIDP; were as pure ST affection was not seen in this illness.

3- Sexual disturbance in males while urinary disturbance in females were the most common autonomic symptoms encountered.

4- A simple test [VM, HR response to standing] that can be performed in outpatient setting allowing an early detection of autonomic dysfunction in those patients even in asymptomatic individuals

\section{Recommendations}

- Proper attention to the assessment and management of autonomic function in CIDP may help in improving the quality of life of those patients; as those disturbances are disabling and in most of the times, simple measures might be taken to overcome such impairment, thus the practicing clinician should be encouraged to look carefully for the autonomic symptoms when they assess their CIDP patients and not merely emphasize on usual CIDP features; because most of those symptoms were disabling and interfered with daily social life.

- Cardiovascular dysfunction may be considered a prognostic factor in the course of CIDP; a prospective study should be performed in order to determine its prognostic implication.

- The need for a special instrument designed to help the patient and the physician to overcome disabilities issues that are encountered in any chronic neurological disease like CIDP.

\section{Appendix}

\section{Appendix 1}

Protocol paper

Autonomic assessment in CIDP

-Patient name ............... Age ........ Sex.........

-Date...

-History:

-Initial symptoms

-chief complaint and duration of illness:

-HOPI:

-Autonomic symptoms:

1. CV disturbance: 4. sexual disturbance:
a. postural dizziness a. impotence
b. syncope b. impaired ejaculation
c. palpitation c. loss of libido
d. flushing sensation d. loss of early morning
2. Urinary disturbance:erection
a. urgency
b. hesitancy 
5. Sweating disturbance:
a. dry skin
b. excessive sweating
c. frequency
d. incontinence
e. nocturia

3. GIT disturbance:
a. constipation
b. faecal incontinence
c. dyspepsia
d. dry mouth

-Drug history and toxic exposure:

-Past medical history:

-Physical examination:

-General medical examination

-Medical examination:

-Neurological examination:

-Work up:

1. general:

-CBP and ESR CXR abd/pelvic US

-ANA VDRL blood biochemistry TFT

2.CSF study:

3.Electrophysiological studies:

4.Autonomic function test:

\section{Appendix 2}

The five standard cardiovascular Ewing tests used forassessment of cardiovascular autonomic neuropathy

A-Tests to detect parasympathetic involvement:

1- Heart rate response to valsalva maneuver: the test is performedby asking the patient to blow into a mouth-piece connected to amodified sphygmo-manometer and holding it at a pressure of $40 \mathrm{mmHg}$ for 15 seconds, while continues electrocardiogram isrecorded. The result are expressed as the valsalva ratio whichthe ratio of the longest $R-R$ interval after the maneuver to theshortest $\mathrm{R}-\mathrm{R}$ interval during the maneuver. The mean of the 3valsalva ratios is taken as the final value.

2- Heart rate (R-R interval) variation during deep breathing: thepatient sits quietly and breathes deeply and even at six breathsper minutes, with 5 seconds allocated for each inspiration andexpiration to optimize the sinus arrhythmia. Anelectrocardiogram is recorded throughout the period of deepbreathing, with a marker to indicate the onset of eachinspiration and expiration. The result is then expressed as themean difference between maximum and minimum heart rate forsix measured cycles in beats per minutes.

3- Immediate heart rate response to standing:the tests isperformed with the patient lying quietly on a couch while theheart rate is recorded continuously on an electrocardiogram.

The shotest R-R interval at or around the 15 th beat and thelongest R-R interval at or around the 30th beat after starting tostand are measured. The characteristic heart rate response isexpressed by the 30:15 ratio (R-R interval of the 30 th to the R-Rinterval of the 15 th ).

B-Tests to detect sympathetic involvement:
1- Blood pressure response to standing: the test is performed bymeasuring the patient blood pressure with sphygmomanometerwhile he is lying down, and again when he stand up for threeminutes. The postural fall of blood pressure is taken as thedifference between systolic blood pressure lying and systolicblood pressure standing.

2- Blood pressure response to sustained handgrip: heresphygmomanometer cuff is used. Handgrip is then maintainedat the $30 \%$ of that maximum for as long as possible up to fiveminutes. blood pressure is measured three times before and atone-minute interval during handgrip. The result was expressedas the difference between the highest diastolic blood pressureduring handgrip exercise and the mean of the three diastolicblood pressure readings before handgrip begun.

The scoring of the five cardiovascular Ewing tests as normal,borderline and abnormal results

Test Normal Borderline Abnormal

Heart rate response to valsalva maneuver $>1.201 .11-1.20$ $<1.10$

Heart rate variation during deep breathing $>15 \mathrm{~b} / \mathrm{m} 11-14$ $\mathrm{b} / \mathrm{m}<10 \mathrm{~b} / \mathrm{m}$

Immediate heart rate response to standing $>1.041 .01-1.03$ $<1.00$

Blood pressure response to standing $<10 \mathrm{mmHg} 11-29$ $\mathrm{mmHg}>30 \mathrm{mmHg}$

Blood pressure response to sustained handgrip $16 \mathrm{mmHg}$ $11-15 \mathrm{mmHg}<10 \mathrm{mmHg}$

\section{References}

[1] Barohn RJ, Saperstein DS: Guillain-Barre syndrome and chronic inflammatory demyelinating polyneuropathy. SeminNeurol 1998; 18(1):49-61.

[2] Dalakas MC, Engel WK. Chronic relapsing (dysimmune) polyneuropathy: pathogenesis and treatment. Ann Neurol 1981;9:Suppl: 134-45.

[3] Barohn RJ, Kissel JT, Warmolts JR, MendellJR. Chronic inflammatory demyelinating polyradiculoneuropathy: clinical characteristics, course, and recommendations for diagnostic criteria. Arch Neurol 1989; 46: 878-84.

[4] Research criteria for diagnosis of chronic inflammatory demyelinating polyneuropathy (CIDP): report from an ad hoc subcommittee of the American Academy of Neurology AIDS Task Force. Neurology 1991; 41:617-8.

[5] Katz JS, Saperstein DS, Gronseth G, Amato AA, Barohn RJ. Distal acquired demyelinating symmetric neuropathy. Neurology 2000; 54:615-20.

[6] Mygland A, Monstad P. Chronic acquired demyelinating symmetric polyneuropathy classified by pattern of weakness. Arch Neurol 2003; 60:260-4.

[7] Federico P, Zochodne DW, Hahn AF, Brown WF, Feasby TE. Multifocal motor neuropathy improved by IVIg: randomized, double- blind, placebo-controlled study. Neurology 2000; 55:1256-62.

[8] Pestronk A. Multifocal motor neuropathy: diagnosis and treatment. Neurology 1998; 51: Suppl 5:S22-S24. 
[9] Oh SJ, Claussen GC, Kim DS. Motor and sensory demyelinating mononeuropathy multiplex (multifocal motor and sensory demyelinating neuropathy): a separate entity or a variant of chronic inflammatory demyelinating polyneuropathy? J PeripherNervSyst 1997; 2:362-9.

[10] Lewis RA, Sumner AJ, Brown AJ, Asbury AK. Multifocal demyelinating neuropathy with persistent conduction block. Neurology 1982; 32:958-64.

[11] Viala K, Renie L, Maisonobe T, et al. Follow- up study and response to treatment in 23 patients with Lewis-Sumner syndrome. Brain 2004; 127:2010-7.

[12] Alaedini A, Sander HW, Hays AP, Latov N. Antiganglioside antibodies in multifocal acquired sensory and motor neuropathy. Arch Neurol 2003; 60:42-6.

[13] Thomas HM. Recurrent polyneuritis: a clinical lecture. Phil Med J. 1898; 1:885-889.

[14] Harris W, Newcomb WD. A case of relapsing interstitial hypertrophic polyneuritis. Brain. 1929; 52:108-116.

[15] Denny-Brown D, Brenner C. Paralysis of nerve induced by direct pressure and by tourniquet. Arch Neurol Psychiatry. 1944;51:1-26.

[16] Dyck PJ, Gutrecht JA, Baston JA, Karnes WE, Dale AJ. Histologic and teased-fiber measurements of sural nerve in disorders of lower motor and primary sensory neurons. Mayo Clin Proc. 1968; 43: 81-123.

[17] Dyck PJ, Lais AC, Ohta M, Bastron JA, Okazaki H, Groover RV. Chronic inflammatory polyradiculoneuropathy. Mayo Clin Proc. 1975; 50:621- 637

[18] Austin JH. Recurrent polyneuropathiesand their corticosteroid treatment; with five year observations of a placebo-controlled case treated with corticotrophin, cortisone, and prednisone. Brain 1958; 81:157-92.

[19] Connolly AM. Chronic inflammatory demyelinating polyneuropathy in childhood. PediatrNeurol 2001; 24:17782.

[20] McLeod JG, Pollard JD, Macaskill P, Mohamed A, Spring P, Khurana V. Prevalence of chronic inflammatory demyelinating polyneuropathy in New South Wales, Australia. Ann Neurol 1999; 46:910-3.

[21] Lunn MP, Manji H, Choudhary PP, Hughes RA, Thomas PK. Chronic inflammatory demyelinating polyradiculoneuropathy: a prevalence study in south east England. J NeurolNeurosurg Psychiatry 1999;66:677-80.

[22] Mygland A, Monstadt P. Chronic polyneuropathies in VestAgder, Norway. Eur J Neurol. 2001; 8: 157-165.

[23] Mohamed A, Pollard J, Walsh JC, et al. Prevalence of CIDP in NSW. J ClinNeurosci 1997; 4:403.

[24] Korinthenberg R: Chronic inflammatory demyelinating polyradiculoneuropathy in children and their response to treatment. Neuropediatrics 1999 Aug; 30(4): 190-6.

[25] Bouchard C, Lacroix C, Plante V, et al: Clinicopathologic findings and prognosis of chronic inflammatory demyelinating polyneuropathy. Neurology 1999 Feb; 52(3): 498-503.
[26] Sander HW, Latov N. Research criteria for defining patients with CIDP. Neurology 2003; 60: Suppl 3:S8-S15.

[27] Gorson KC, Allam G, Ropper AH. Chronic inflammatory demyelinating polyneuropathy: clinical features and response to treatment in 67 consecutive patients with and without a monoclonal gammopathy. Neurology 1997;48:321-8.

[28] Gorson KC, Ropper AH, Adelman LS, Weinberg DH. Influence of diabetes mellitus on chronic inflammatory demyelinating polyneuropathy. Muscle Nerve 2000; 23:37-43.

[29] Haq RU, Pendlebury WW, Fries TJ, Tandan R. Chronic inflammatory demyelinating polyradiculoneuropathy in diabetic patients. Muscle Nerve 2003; 27:465-70.

[30] Ginsberg L, Malik O, Kenton AR, et al. Coexistent hereditary and inflammatory neuropathy. Brain 2004; 127:193-202.

[31] Saperstein DS, Katz JS, Amato AA, Barohn RJ. Clinical spectrum of chronic acquired demyelinating polyneuropathies. Muscle Nerve 2001; 24:311-24.

[32] Hughes R, Bensa S, Willison H, et al. Randomized controlled trial of intravenous immunoglobulin versus oral prednisolone in chronic inflammatory demyelinating polyradiculoneuropathy. Ann Neurol 2001; 50: 195-201.

[33] Mathias CJ.Autonomic disorders and their recognition. The N Engl J Med 1997; 336:721-4.

[34] Rogelio MG. Central autonomic regulation; Primer on ANS. In: David R, Low PAL, Polinsky RJ eds. California, Academic Press, 1996; 1: 3-12.

[35] Engstrom J, Martin JR. Disorders of ANS. In : Isselbacher KJ, Brawn WE, Wilson ID, Martine JB, Fauci AS, Kasper DI eds. Harrison s Principles of Internal Medicine, 14th ed. New York, Mc-Graw-Hill 1998; 371:2372-8.

[36] Drovy V E , Nisipeanu P F, Kroczyn A D. Tests of autonomic dysfunction in patients with multiple sclerosis. Acta Neurol Scand 1995; 92(5):356-60.

[37] Bebarroch E E. CNS Disorders. In: Robertson D, Low Ph A, Polinsky R J eds. Primer on ANS. California, Academic Press, 1996; 9: 226-30.

[38] Landsbery L, Young JR. Physiology and pharmacology ANS. In:Isselbacher KJ, Brawn WE, Wilson ID, Martine JB, Fauci AS, Kasper DI eds. Harrison s Principles of Internal Medicine, 14th ed. New York, Mc-Graw-Hill 1998; 70: 430-1.

[39] Macleod JG, Tuck RR. Disorders of ANS: Part 1; Pathophysiology and clinical features. Ann Neurol 1987; 21:114-30.

[40] Macleod JG, Tuck RR. Disorders of ANS: Part 2; Investigation and Treatment. Ann Neurol 1987; 21:114-30.

[41] Ewing DJ, Clarke BF. Diagnosis and management of diabetic autonomic neuropathy. Brit Med J 1982; 285: 915-8.

[42] ]Pfeifer M. Cardiovascular assessment. In: Dyck PJ, Thomas PK eds. Diabetic neuropathy, 2nd ed., 1999; 11: 171-83.

[43] Low PA, Fealey RD. Sudomotor neuropathy. In: Dyck PJ, Thomas PK eds. Diabetic neuropathy, 2nd ed., 1999; 13:191-9. 